\title{
Clinical inertia on insulin treatment in the primary care setting
}

Felipe Vázquez, ${ }^{1}$ Pilar Lavielle, ${ }^{2 *}$ Rita Gómez-Díaz ${ }^{2}$ and Niels Wacher ${ }^{2}$

${ }^{1}$ Secretaria de Salud, Psychiatric Health Services; 'Instituto Mexicano del Seguro Social, Centro Médico Nacional Siglo XXI, Specialty Hospital, Clinical Epidemiology Research Unit. Mexico City, Mexico

\begin{abstract}
Introduction: Refusal of physicians to prescribe insulin to their patients has been scarcely evaluated; the delay in treatment intensification hinders adequate and quality care. Objective: To identify the perception of primary care physicians about barriers to initiate insulin treatment in patients with diabetes. Method: Using the Smith Index and multivariate analysis, the relevance and grouping of concepts related to barriers to insulin prescription were assessed in 81 family doctors. Results: Only $35.8 \%$ of physicians showed confidence for prescribing insulin; almost half of them rated treatment intensification between moderately and little important (39.5\% and $6.2 \%$ ). Barriers were related to the physician (39.5\%), the patient (37\%), insulin treatment (11.1\%) and the institution (6.2\%); $6.2 \%$ of physicians did not perceive any barrier. The barriers were grouped in 5 factors that explained $62.48 \%$ of the variance: patient cultural level, lack of medical skills, fear of adverse events, insecurity and lack of training. Conclusion: Clinical inertia was not the result of a complex medical condition or patient comorbidities, but of doctor's perception and confidence in his/her clinical and communication skills.
\end{abstract}

KEY WORDS: Clinical inertia. Primary care. Insulin treatment. Barriers.

\section{Introduction}

The prevention and treatment of type 2 diabetes (DM2) has become one of the main challenges for health institutions. Global prevalence of this condition has increased from $4.7 \%$ in 1980 to $8.5 \%$ in $2014 .{ }^{1}$ In Europe, the prevalence of diabetes has been estimated to be $7.8 \%$; in North America and the Caribbean it is $11.4 \%$, and in South America, $8.1 \% .^{2}$ In Mexico, the results of the half-way 2016 National Health and Nutrition Survey (ENSANUT - Encuesta Nacional de Salud y Nutrición) showed that the prevalence of known diabetes cases was $9.2 \%{ }^{3}$ This survey also reported that only $25.3 \%$ of patients reached the glycosylated hemoglobin ( $\mathrm{HbA1c}$ ) therapeutic goals of $<7 \%$ and only 13 $\%$ of those receiving medical care were treated with insulin for the control of hyperglycemia. ${ }^{4}$
Numerous patients with diabetes initially treated with diet, exercise or medication will eventually require treatment intensification. ${ }^{5}$ However, it has been demonstrated that this therapeutic measure can be delayed for several years, particularly when insulin is the next treatment option. ${ }^{6}$ Different studies have focused on patients' "psychological insulin resistance", a term that refers to their refusal to accept insulin treatment due to negative expectations about the results: $;, 8$ however, few evaluate physicians' rejection to prescribe insulin. ${ }^{9}$ This phenomenon -known as "clinical inertia"- is characterized by the inability of the physician to intensify treatment in a timely manner, a delay that is one of the most important factors that hinder adequate and quality care for patients with DM2 (i.e., correcting poor glycemic control). ${ }^{10}$ According to published studies, clinical inertia can affect
Gac Med Mex. 2019;155:143-147

Contents available at PubMed www.gacetamedicademexico.com 
between 30 and $68 \%$ of cases, a proportion that can be increased when $\mathrm{HbA1c}$ is higher than $9 \% .{ }^{11}$

Given that clinical inertia is primarily the responsibility of the physician, ${ }^{12}$ and that behavioral change requires for the individual to perceive its importance and feel confident about that which he/she has to do (self-efficacy), the present study aimed at identifying the perception of the family physician about the importance, barriers and confidence with regard to starting insulin treatment in DM2-diagnosed patients. This knowledge can be of great help for implementing appropriate interventions to support the family physician to properly use insulin treatment and, as a consequence, to increase the proportion of patients that achieve the therapeutic goals regarding $\mathrm{HbA} 1 \mathrm{c}$ levels.

\section{Method}

To ensure the validity of data, a mixed study was carried out (quantitative surveys that were combined with qualitative methods).

A purpositive, convenience sample of 81 family doctors who attended the Training Center of the Mexican Institute of Social Security was invited to participate in a course on DM2 aimed at increasing the quality of care of this condition. The course was part of the DiabetIMSS program and the protocol was approved by the Research Committee of the institution (IMSS2004-131). Doctors gave their verbal consent to participate after the objectives and dynamics of the study were explained.

Semi-structured interviews were conducted to understand the doctors' perception of the barriers to starting insulin treatment. ${ }^{13}$ In a card, the doctors answered a question (anonymously) that assessed their perception on the barriers they faced in daily clinical practice to initiate insulin therapy in patients with DM2. Using a 10-point visual analogue scale (where 0 was the lowest score), the physicians rated the degree of importance and confidence they experienced for prescribing insulin.

The interviews were evaluated through content or coding analysis (qualitative information reduction process) to obtain qualitative and quantitative data. Content analysis is a research methodology based on standardized procedures that focuses on the study of contents and its objective is a systematic, valid and reproducible description of communication or any other behavioral manifestation. The meaning of words, topics or phrases is the information that is quantified.
Based on this technique, the concepts extracted from the interviews were classified into a certain number of categories according to their semantic similarity, which reflected the cognitive patterns of the study group. This procedure allowed constructing a two-way matrix (participant/category) and performing a quantitative analysis to calculate the frequencies of the concepts mentioned by the participants in the interviews. ${ }^{14}$

Subsequently, the Smith Index or salience score was calculated to determine which category of barriers to insulin prescription was the most relevant. The calculation of the preponderance index is a cognitive anthropology technique -used in multiple scientific disciplines- to describe and analyze the participants' culture and behaviors from their point of view. This index is calculated based on two criteria:

- The concepts mentioned first.

- The most frequently mentioned concepts.

Based on this index, a description is made of what people think about a particular phenomenon and the way they organize this information, in order to determine the most important concepts for the respondents in relation to the investigated subject, in this case, barriers to prescribing insulin. 15,16 The most important advantage of this technique is that it yields in-depth information about subjects' cognitive process through a method that is easy to apply and understand by participants. Only the concepts mentioned with a frequency $\geq 4$ were included, since those concepts referred to less frequently were not shared by the group..$^{17}$

Finally, according to the purposes of the study and the type of generated data, a multivariate analysis of the main components was carried out to describe the relationship between concepts, ${ }^{18,19}$ with those that were grouped within the same factor (according to the Kaiser criteria, the factors whose associated eigenvalue was greater than 1 were preserved) being examined.

\section{Results}

Out of 81 interviewed family physicians, $22.7 \%$ were females. Mean age was 45 years. Average years of experience as family physicians was 15 years.

Most participating physicians reported low levels of confidence in their abilities to start insulin treatment in patients with diabetes: $14.8 \%$ admitted not feeling confident in their abilities, $49.4 \%$ felt moderately confident and only $35.8 \%$ were confident for providing this type of treatment. A surprising finding was 
that almost half the family doctors rated treatment intensification in patients with diabetes between moderately important and unimportant $(39.5 \%$ and $6.2 \%$, respectively).

The analysis of the interviews showed that the barriers to initiate insulin treatment were related to characteristics of the doctor in $39.5 \%$, the patient in 37 $\%$ and treatment in $11.1 \%$. Only $6.2 \%$ of family doctors did not perceive any barrier to start insulin in their patients and referred already prescribing it in those who required it, with positive results. Finally, $6.2 \%$ considered that the institution was also a barrier to prescription.

According to the Smith Index (Table 1), the main barriers perceived by family doctors to prescribe insulin were patients' cultural beliefs and physicians' lack of training, followed by patient refusal to receive the treatment and insulin adverse effects, such as hypoglycemia.

With the nine categories shared by physicians about barriers to prescribing insulin, a multivariate analysis of the main components was carried out to determine the relationship between them. According to the analysis, the categories were grouped in five factors (which reached eigenvalues higher than 1 according to the Kaiser criterion), as shown in Table 2. These five factors explained $62.48 \%$ of variance: factor 1 , $15.4 \%$; factor 2, $13.3 \%$; factor $3,12.3 \%$; factor 4 , $10.9 \%$; factor $5,10.4 \%$ :

\section{Factor I}

This factor was integrated by patients' cultural and cognitive aspects that doctors considered as the most important barriers to insulin prescription. The main concepts that were referred were "patient myths about insulin", "patient taboos", "patient cultural beliefs that prevent them from accepting the treatment", "stigma", "poor acceptance of insulin by patients", "patient beliefs that insulin can make them blind".

\section{Factor II}

This factor comprises physicians' negative attitudes and behaviors that increase patient rejection towards insulin treatment. It includes barriers reported by physicians about their lack of experience in the management of insulin, as well as a deficiency in communication skills that has prevented them from adequately presenting insulin treatment cost-benefit balance to the patient: "I have not enough experience", "I have
Table 1. Main categories mentioned by family doctors about barriers to prescribing insulin, according to the Smith Index (order and frequency of mention)

\begin{tabular}{|l|r|c|c|}
\hline Category & $\mathrm{n}$ & Answer \%* & Smith Index \\
\hline Patient cultural limitations & 16 & 19.75 & 0.28 \\
\hline Patient refusal & 12 & 14.81 & 0.27 \\
\hline Hypoglycemia & 12 & 14.81 & 0.25 \\
\hline Physician training & 14 & 17.28 & 0.18 \\
\hline Patient adherence & 9 & 11.11 & 0.18 \\
\hline Medical inexperience & 8 & 9.87 & 0.18 \\
\hline $\begin{array}{l}\text { Physician lack of communication } \\
\text { skills }\end{array}$ & 6 & 7.40 & 0.1 \\
\hline $\begin{array}{l}\text { Physician lack of confidence } \\
\text { Patient ignorance }\end{array}$ & 5 & 6.17 & 0.1 \\
\hline *Percentage of physicians who answered to the indicated category. &
\end{tabular}

not done it before", "I am unable to convince the patient", "I do not have the capacity to present adequate information in order to convince the patient", "we must increase patient awareness and trust".

\section{Factor III}

Doctors reported that one of the barriers to prescribing insulin is fear of medication-related adverse effects (hypoglycemia). In this factor, physicians' own fears stand out, from causing hypoglycemia or being criticized by other doctors, to the fear of causing patient death: "applying a dose that might cause hypoglycemia", "since I've never used it, I am afraid that the patient may experience hypoglycemia without anybody noticing".

\section{Factor IV}

Physicians reported their feelings of insecurity with regard to the management of insulin: "insecurity", "lack of confidence in the management of insulin", "lack of confidence in prescribing nocturnal insulin". Some doctors are not motivated to prescribe insulin and preferred referring the patient to the endocrinologist.

\section{Factor $V$}

This factor included physicians' poor training for the management of insulin and for identifying appropriate candidates to receive it: "lack of training", "learning 
Table 2. Grouping of concepts referred by family doctors about barriers to prescribing insulin, according to factor analysis

\begin{tabular}{|c|c|c|c|c|c|}
\hline Concept & $\begin{array}{c}\text { Factor } \\
\text { I }\end{array}$ & $\begin{array}{c}\text { Factor } \\
\text { II }\end{array}$ & $\begin{array}{c}\text { Factor } \\
\text { III }\end{array}$ & $\begin{array}{c}\text { Factor } \\
\text { IV }\end{array}$ & $\begin{array}{l}\text { Factor } \\
\text { V }\end{array}$ \\
\hline Patient ignorance & 0.648 & & & & \\
\hline $\begin{array}{l}\text { Patient cultural } \\
\text { beliefs }\end{array}$ & 0.624 & & & & \\
\hline Medical inexperience & & 0.407 & & & \\
\hline $\begin{array}{l}\text { Physician lack of } \\
\text { communication skills }\end{array}$ & & 0.534 & & & \\
\hline Patient refusal & & 0.480 & & & \\
\hline Hypoglycemia & & & 0.643 & & \\
\hline Non-adherence & & & 0.285 & & \\
\hline Physician insecurity & & & & 0.859 & \\
\hline Medical training & & & & & 0.310 \\
\hline
\end{tabular}

who the right patients for insulin treatment are and how to use it".

\section{Barriers related to the health institution}

The health institution was not one of the aspects that were commonly reported; however, it is pertinent mentioning that some physicians referred the difficulties to obtain insulin in the pharmacy and inadequate professional relationship with other specialists in general hospitals.

\section{Discussion}

This study provides important information about a wide range of beliefs and attitudes of physicians about intensifying the treatment in patients with DM2. Our study was based on semi-structured interviews with primary care physicians who participated in a course on diabetes to improve the quality of care. This type of sample and methodological approach allowed through physicians' reflections- to know personal feelings and beliefs about the experience with insulin prescription and to understand the clinical inertia phenomenon.

We identified that the five main causes of clinical inertia perceived by physicians were patients' cultural background, poor medical skills, fear of insulin-related adverse events, medical insecurity and lack of training.

For the physicians of our study, the main factor that explains clinical inertia was patients' rejection to insulin treatment, based on cultural beliefs such as taboos and myths about insulin, on feeling stigmatized, as well as on cognitive processes that generated negative attributions and emotional factors, such as fear. However, this appreciation is not accurate. In other investigations, physicians have been reported to a priori estimate the probability of patient rejection to treatment intensification without having asked them before making a decision, and therefore it is not clear how accurate doctors' estimates about patient preferences or self-management skills are. ${ }^{20}$ For example, it has been estimated that $30 \%$ of patients who do not adhere to treatment indicated being afraid of injections, while $97 \%$ of doctors considered this was the main cause for lack of adherence. ${ }^{21}$ In contrast, doctors from other specialties (endocrinology or internal medicine) do not think this is a barrier to insulin treatment. The difference in doctors' perception about patient barriers may reflect the resistance of family physicians to prescribe insulin. ${ }^{20,22}$

These data are related to another finding: insulin prescription increases stress in family physicians (a phenomenon barely explored in previous studies on clinical inertia). Participants in this study recognized feelings of insecurity, lack of experience in prescribing insulin and fear of possible adverse effects. ${ }^{23,24}$ These negative feelings were related to the perception that poor training and communication skills are the main impediment to presenting an adequate cost-benefit balance, which in turn leads to the patient refusing to start insulin treatment. These results are consistent with those of other studies, where insulin prescription was found to generate high levels of anxiety in family doctors, whose emotions may be one of the main reasons for clinical inertia.

Several investigations have shown the negative consequences these emotional factors can produce. On one hand, a behavior that is too cautious in clinical practice, reduces patient access to specific procedures (such as insulin prescription), thus affecting the quality of medical care. ${ }^{25}$ On the other hand, when perceiving doctor's insecurity, patients may consider insulin prescription as an incorrect medical decision, especially if they perceive negative attitudes in physicians, such as the threat to initiate insulin treatment as a punishment in case of lack of adherence. ${ }^{26}$ These factors can play a very important role in patient acceptance of insulin treatment and become a barrier in metabolic control. ${ }^{27}$

As the results of our research suggest, it is important to increase family physician's confidence and security in his/her clinical skills for prescribing insulin to 
patients with DM2, through training programs in this area, which should also include psychological and communication strategies. This can positively influence on the way doctor's instructions are understood and remembered, on patient clinical outcomes and on the quality of care. ${ }^{28,29}$

This study has some limitations that are important to be taken into account. The used sample might lead to an overestimation of the results: since the interviewed doctors were participating in a course to improve the care of patients with diabetes, it is possible that they perceived some deficiencies in this area, an appreciation that may not be shared by other family doctors. Therefore, it is important to conduct a study with a representative sample of family doctors to corroborate the results.

\section{Conclusions}

Regardless of physician's considerations about the importance of insulin treatment, clinical inertia was not the result of a complex clinical condition or patient comorbidities, but of feelings and beliefs of family physicians about patient behavior and self-confidence in their clinical and communication skills, which affects the quality of the care they provide.

\section{Acknowledgements}

The authors thank Susan Drier Jonas for her support with the manuscript.

\section{References}

1. World Health Organization. Global status report on non-communicable diseases. Ginebra, Suiza: World Health Organization; 2014.

2. International Diabetes Federation. IDF Diabetes Atlas. Octava edición. Bélgica: International Diabetes Federation; 2018.

3. Hernández-Ávila M, Rivera-Dommarco J, Shamah-Levy T, Cuevas-Nasu L, Gómez-Acosta LM, Gaona-Pineda EB, et al. Encuesta Nacional de Salud y Nutrición de Medio Camino 2016. Informe final de resultados. México: Secretaría de Salud/Instituto Nacional de Salud Pública; 2016.

4. Hernández-Ávila M, Gutiérrez JP, Reynoso-Noverón N. Diabetes mellitus in Mexico. Status of the epidemic. Salud Publica Mex. 2013;55:S129-S136.

5. Barag SH. Insulin therapy for management of type 2 diabetes mellitus: strategies for initiation and long-term patient adherence. J Am Osteopath Assoc. 2011;111:S13-S19.
6. Brath $\mathrm{H}$, Paldánius $\mathrm{P}$, Bader G, Kolaczynski WM, Nilsson P. Differences in glycemic control across world regions: a post-hoc analysis in patients with type 2 diabetes mellitus on dual antidiabetes drug therapy. Nutr Diabetes. 2016;6:e217.

7. Hermanns N, Mahr M, Kulzer B, Skovlund SE, Haak T. Barriers towards insulin therapy in type 2 diabetic patients: results of an observational longitudinal study. Health Qual Life Outcomes. 2010;8:113.

8. Ali A, Cheng AYY, Yu CH. Breaking down barriers to initiating insulin. Insulin prescription pad. Can Fam Physician. 2015;61:446-447.

9. Brown JB, Nichols GA, Perry A. The burden of treatment failure in type 2 diabetes. Diabetes Care. 2004;27:1535-1540.

10. Khunti K, Wolden ML, Thorsted BL, Andersen M, Davies MJ. Clinical inertia in people with type 2 diabetes: a retrospective cohort study of more than 80,000 people. Diabetes Care. 2013;36:3411-3417.

11. Harris SB, Kapor J, Lank CN, Willian AR, Houston T. Clinical inertia in patients with T2DM requiring insulin in family practice. Can Fam Physician. 2010;56:e418-e424

12. O'Connor PJ. Improving diabetes care by combating clinical inertia. Health Serv Res. 2005:40:1854-1861.

13. Willms D, Johnson N. Essentials in qualitative research: a notebook for the field. Canada: McMaster University; 1996.

14. Krippendorff K. Content analysis: an introduction to its methodology. EE. UU.: Sage Publications Beverly Hills; 1980.

15. Smith JJ, Borgatti SP. Salience counts - and so does accuracy: correcting and updating a measure for free-list-item salience. J Linguistic Anthropol. 1998;7:208-209.

16. Thompson EC, Juan Z. Comparative cultural salience: measures using free-list data. Field Methods. 2006;18:398-412.

17. Romney AK, Weller SC, Batchelder WH. Culture as consensus: a theory of cultural and informant accuracy. Am Anthropol. 1986;88:313-338.

18. DeVellis RF. Scale development. Theory and applications. EE. UU.: Sage Publications; 1991.

19. Nunnally JC. Psychometric theory. EE. UU.: McGraw-Hill; 1978.

20. O'Connor PJ, Sperl-Hillen JA, Johnson PE, Rush WA, Biltz G. Clinical inertia and outpatient medical errors. En: Henriksen K, Battles J, Marks $E$, Lewin D, editores. Advances in patient safety: from research to implementation. EE. UU.: Agency for Healthcare Research and Quality; 2005.

21. Ratanawongsa N, Crosson JC, Schillinger D, Karter AJ, Saha CK, Marrero DG. Getting under the skin of clinical inertia in insulin initiation: the Translating Research Into Action for Diabetes (TRIAD) Insulin Starts Project. Diabetes Educ. 2012;38:94-100.

22. Escalada J, Orozco-Beltran D, Morillas C, Alvarez-Guisasola F, Gomez-Peralta F, Mata-Cases M, et al. Attitudes towards insulin initiation in type 2 diabetes patients among healthcare providers: a survey research. Diabetes Res Clin Pract. 2016;122:46-53.

23. García-Morales G, Reyes-Jiménez M. Inercia clínica en pacientes con diabetes mellitus tipo 2 en una unidad de medicina familiar de Acapulco, Guerrero, México. Aten Fam. 2017;24:102-106.

24. Lerman I. Barreras que dificultan la aplicación temprana de insulina en el paciente con diabetes tipo 2. ALAD. 2009;17:66-68.

25. Alkhaifi M, Al Khusaibi GA, Theodorson T, Ward MA, Mazrou'l AA. Barriers in initiating insulin treatment in type 2 diabetes mellitus among physicians in Wilayat of Bowsher in Oman. J Fam Med Community Health. 2015;2:1034.

26. Gherman A, Veresiu IA, Sassu RA, Schnur JB, Scheckner BL, Montgomery GH. Psychological insulin resistance: a critical review of the literature. Diabetes Int. 2011;28:125-128.

27. Lee $\mathrm{Y}$, Lee $\mathrm{P}, \mathrm{Ng}$ CJ. A qualitative study on healthcare professionals perceived barriers to insulin initiation in a multi-ethnic population. BMC Fam Pract. 2012;13:28.

28. Owens D. Clinical evidence for the earlier initiation of insulin therapy in type 2 diabetes. Diabetes Technol Ther. 2013:15:776-785.

29. The NHS Atlas of Variation in Healthcare. Reducing unwarranted variation to increase value and improve quality. Inglaterra: Public Health England/NHS Englad/Care Quality Commission/Health and Social Care Information Centre/Office for National Statistics/NHS Improving Quality; 2016. 